\title{
HONGOS ANAMORFOS ASOCIADOS A RESTOS VEGETALES DEL PARQUE ESTATAL “AGUA BLANCA”, MACUSPANA, TABASCO, MÉXICO
}

\author{
Karen Martínez-Rivera ${ }^{1,3}$, Gabriela Heredia ${ }^{2}$, Edmundo Rosique-GiL ${ }^{1}$ \\ Y Silvia CAPPEllo ${ }^{1}$
}

${ }^{1}$ Universidad Juárez Autónoma de Tabasco, División Académica de Ciencias Biológicas, Laboratorio de Micología, Herbario UJAT, km 5 carretera Villahermosa - Cárdenas entronque a Bosques de Saloya, 86039 Villahermosa, Tabasco, México.

${ }^{2}$ Instituto de Ecología, A.C., km 2.5 antigua carretera a Coatepec 351, Congregación El Haya, 91070 Xalapa, Veracruz, México.

${ }^{3}$ Autor para la correspondencia: k_martinezrivera@hotmail.com

\section{RESUMEN}

La riqueza fúngica de las áreas protegidas del estado de Tabasco ha sido pobremente estudiada. En su mayoría, las especies saprobias microscópicas aún se desconocen. La presente contribución tiene como objetivo documentar la composición de hongos anamorfos que proliferan en los restos vegetales de la selva del Parque Estatal "Agua Blanca". En total se presentan 40 taxones, entre los cuales se incluyen 15 que no se conocían para México y 25 para el estado de Tabasco. De todos los registros nuevos a nivel nacional, junto con su descripción morfológica, se anexan ilustraciones, información sobre la distribución geográfica conocida y observaciones sobre sus caracteres de interés taxonómico.

Palabras clave: hongos microscópicos, hongos saprobios, hongos tropicales, restos vegetales.

\section{ABSTRACT}

Fungal diversity of protected areas in the state of Tabasco has been poorly studied. Most microscopic saprophytic species are not yet known. This contribution aims to increase our knowledge of the litter-inhabiting anamorphic fungi from the rain forest of the "Agua Blanca" state park. An account of 40 taxa is included, 15 of them had not been reported 
from Mexico before, and 25 are new records for the Tabasco state. For all the new national records, morphologic description, illustrations, geographical distribution and observations on their taxonomic characters are included.

Key words: microfungi, plant debris, saprobe fungi, tropical fungi.

\section{INTRODUCCIÓN}

Más de $60 \%$ de las especies del reino Fungi son microscópicas, representando un grupo con una amplia distribución tanto en hábitats naturales como en los ambientes modificados por el hombre (Heredia et al., 2008). Entre ellas destacan los hongos anamorfos por tener patrones de distribución extendidos y por ser abundantes tanto en ambientes naturales como en los domésticos, donde es frecuente encontrarlos como parásitos o saprobios. La mayoría de los estudios sobre estos hongos se han enfocado en los patógenos. Por el contrario, se conoce muy poco sobre la diversidad de las especies saprobias, a pesar de su alto potencial en la biotecnología y su participación en importantes procesos tales como la descomposición de restos vegetales y el reciclaje de nutrimentos (Heredia et al., 2008).

Si bien en la última década se han incrementado las publicaciones sobre la descripción de nuevas especies y registros de anamorfos, para la mayoría de las entidades de la República Mexicana aún no se cuenta con información y mucho menos con colecciones de referencia. Con respecto al estado de Tabasco, Rosique y Cappello (en prensa) mencionan que se han reportado 467 especies de macromicetos y micromicetos, entre las que están 128 taxones de micromicetos anamorfos, correspondientes a organismos saprobios asociados a restos vegetales (Heredia et al., 2006; Becerra et al., 2007, 2008 y 2011).

El estado de Tabasco presenta una considerable riqueza vegetal, por lo cual se puede esperar que exista una gran diversidad de especies de hongos anamorfos en sus ecosistemas; es por eso que es necesaria la realización de proyectos a largo plazo, encaminados a la exploración de las áreas conservadas y protegidas del estado. Tal es el caso del Parque Estatal "Agua Blanca”, localizado en el ejido Las Palomas, del municipio de Macuspana, en la porción oeste y noroeste de la Región

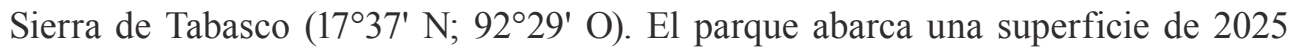
hectáreas (Fig. 1) (Castillo y Zavala, 1996; Anónimo, 2009), con una altitud de 100 a $200 \mathrm{~m}$. La vegetación dominante es selva mediana perennifolia; se han registrado 


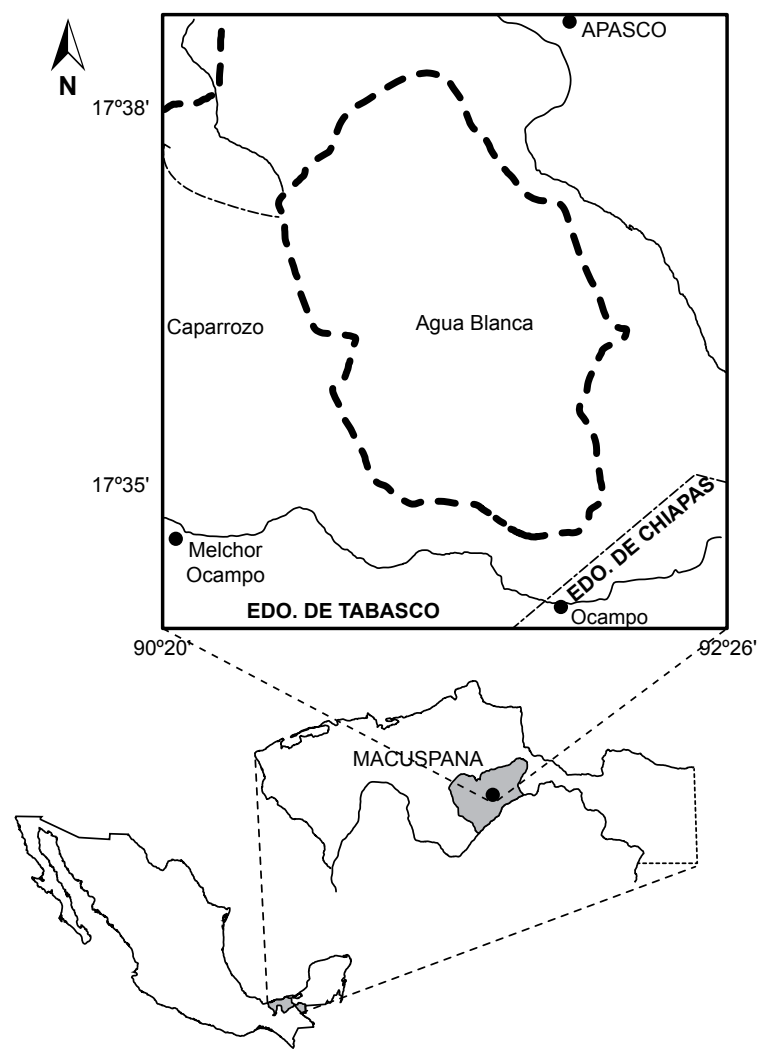

Fig. 1. Ubicación geográfica del Parque Estatal Agua Blanca, Macuspana, Tabasco.

1950 especies de plantas vasculares pertenecientes a 150 familias (Castillo, 1995), que representan $49 \%$ de la diversidad vegetal del estado.

El objetivo de este estudio es contribuir al conocimiento de los hongos anamorfos saprobios del estado de Tabasco, mediante la descripción y documentación de especies recolectadas en restos vegetales de la selva del Parque Estatal "Agua Blanca".

\section{MÉTODO}

En el área de selva mediana del parque se recolectaron del suelo restos vegetales en diferentes estados de descomposición. En el laboratorio, el material se 
colocó en cámaras húmedas a temperatura ambiente. A partir del segundo día de incubación las muestras se revisaron diariamente mediante un microscopio estereoscópico (Zeiss Stemi DV4). Se hicieron preparaciones permanentes con alcohol polivinílico y semipermanentes con lactofenol. El análisis de la morfología, de la conidiogénesis y la obtención de las medidas de las estructuras se efectuaron mediante microscopía de campo claro (Carl Zeiss Axiostar Plus) y de contraste de fases (Nikon-Eclipse 80i). La identificación de las especies se llevó a cabo con la consulta de bibliografía especializada, además de los textos básicos sobre hongos anamorfos (Ellis, 1971, 1976; Carmichael et al., 1980; Barnett y Hunter, 1987; Matsushima, 1971, 1983, 1996 y 2001). La información sobre la distribución geográfica de los hongos se obtuvo mediante la consulta de bibliografía especializada y la base de datos CYBERNOME (www.cybertruffle.org.uk/cybernome/eng). El material de referencia consistente en preparaciones microscópicas se encuentra depositado en la Sección de Micología del Herbario de la Universidad Juárez Autónoma de Tabasco (UJAT).

\section{RESULTADOS}

Se identificaron 40 especies, de las cuales 15 son nuevos registros para México y 25 para el estado de Tabasco. Dado que todas las especies estudiadas han sido recolectadas en diferentes regiones tropicales del mundo, es factible suponer que su distribución es pantropical. De acuerdo con la búsqueda bibliográfica y la consulta de la base de datos CYBERNOME, Dactylaria filiformis, Mycoleptodiscus taiwanensis y Vermiculariopsiella parvula no habían sido vueltas a registrar desde su descripción original. Así mismo, para Dactylaria uniseptata, Melanocephala cupulifera, Mycoleptodiscus taiwanensis y Vermiculariopsiella parvula no se encontraron registros de colectas previas para la zona neotropical por lo que con la presente contribución se extiende el conocimiento de su distribución geográfica.

Descripción morfológica de las especies que representan nuevos registros para México

Atrosetaphiale flagelliformis Matsush., Matsush. Mycol. Mem. 8: 14. 1995. Fig. 2.

Colonias dispersas, pardas oscuras. Conidióforos macronematosos, mononematosos, solitarios o gregarios, erectos, rectos, o ligeramente curvos, simples, lisos, 
pardos oscuros, 73.8-117 × 4.1-5 $\mu \mathrm{m}$. Células conidiógenas enteroblásticas, fialídicas, ampuliformes, integradas en el ápice y agrupadas en verticilos de 2-4 fiálides, dispuestas en intervalos a lo largo de los conidióforos, lisas, pardas pálidas, 9.4-14 $\times$ 4.2-5 $\mu \mathrm{m}$. Conidios filiformes, sin septos, cilíndrico-fusiformes hacia la base y flageliformes hacia el ápice, unicelulares, hialinos, agregados en mucílago, 12-30× $1-1.5 \mu \mathrm{m}$.

Material estudiado. Hojas en descomposición de Aechmea bracteata (Bromeliaceae). CHM-UJAT-01. Col. K. Martínez-Rivera, 11/11/2010.

Observaciones. La forma y dimensiones del ejemplar estudiado coinciden con las del material tipo de la especie.

Distribución. Hasta la fecha Atrosetaphiale es un género monoespecífico, el material tipo fue aislado de pecíolos de palma recolectados en la región amazónica del Perú (Matsushima, 1995). La especie también ha sido recolectada en Brasil (Castañeda-Ruiz et al., 2003; Barbosa et al., 2009).

Beltraniopsis ramosa R.F. Castañeda, Rev. Jardín Bot. Nac. 6(1): 53. 1985. Figs. 3a y 3 b.

Colonias dispersas, pilosas, pardas claras. Conidióforos macronematosos, setiformes, con 1-3 ramificaciones, erectos, ápices afilados, lisos, pardos, $250 \times 3-5$ $\mu \mathrm{m}$. Células conidiógenas poliblásticas, integradas o discretas, denticuladas, lisas, pardas pálidas, 9-13 × 5-5.5 $\mu \mathrm{m}$. Células separadoras ovoides, lisas, hialinas. Conidios bicónicos, rostrados hacia el ápice, base redondeada, oliváceos con una banda transversal hialina, $22-26 \times 5-6 \mu \mathrm{m}$.

Material estudiado. Hojas en descomposición. CHM-UJAT-03. Col. K. Martínez-Rivera, 11/02/2011.

Observaciones. Castañeda-Ruiz y Arnold (1985) mencionan la presencia de conidióforos con paredes verrugosas, carácter que no se observó en el material examinado. Sin embargo, el resto de la morfología coincide con la descripción de $B$. ramosa.

Distribución. Existen registros para Brasil (Gusmão y Grandi, 1996; Gusmão et al., 2001), Cuba (Castañeda-Ruiz y Arnold, 1985; Delgado-Rodríguez y MenaPortales, 2004a; Delgado-Rodríguez et al., 2002) y Venezuela (Castañeda-Ruiz, 2009).

Berkleasmium sinense Joanne E. Taylor, K.D. Hyde \& E.B.G. Jones, Fungal Diversity 12: 302. 2003. Fig. 4. 
Conidiomas de tipo esporodoquio, punctiformes, pardos oscuros, brillosos. Conidióforos micronematosos, simples o ramificados, hialinos, 2-2.5 $\mu \mathrm{m}$. Células conidiógenas holoblásticas, integradas, terminales, hialinas, cilíndricas. Conidios elipsoidales u obovoides, muriformes, lisos, ligeramente constreñidos en los septos, pardos oscuros en el ápice, más claros hacia la base, 30-51 × 21-30 $\mu \mathrm{m}$.

Material estudiado. Ramas en descomposición. CHM-UJAT-04. Col. K. Martínez-Rivera, 11/02/2011.

Observaciones. Las medidas del ejemplar estudiado concuerdan con las del material tipo reportado por Taylor y Hyde (2003).

Distribución. Esta especie ha sido recolectada en sitios tan distantes como Argentina (Capdet y Romero, 2010) y China (Taylor y Hyde, 2003).

Clonostachys compactiuscula (Sacc.) D. Hawksw. \& W. Gams, Trans. Br. Mycol. Soc. 64(1): 90. 1975. Figs. 5a y 5b.

Colonias gregarias, dispersas, algodonosas, mucilaginosas, blancas. Conidióforos macronematosos, mononematosos, flexuosos, en grupos, con el ápice peniciliforme, septados, lisos, hialinos. Células conidiógenas monofialídicas, 2-4, determinadas, lisas, hialinas, 10.5-18 $\times$ 2.5-3 $\mu \mathrm{m}$. Conidios sin septos, cilíndricos, hialinos, agrupados en columnas mucilaginosas, $8-11 \times 1-2 \mu \mathrm{m}$.

Material estudiado. En hojas en descomposición. CHM-UJAT-05. Col. K. Martínez-Rivera, 11/02/2011.

Observaciones. La presencia de conidióforos de tipo Verticillium y la disposición de los conidios en largas columnas mucilaginosas, claramente distinguen a esta especie.

Distribución. Muy probablemente se trata de un hongo cosmopolita asociada a suelos y restos vegetales; existen registros para Argentina (Arambarri et al., 1987), Brasil (Barbosa et al., 2009), Cuba (Mercado-Sierra y Mena-Portales, 1995), Inglaterra (Hawksworth y Punithalingam, 1975) y Venezuela (Castañeda-Ruiz et al., 2003).

Dactylaria filiformis R.F. Castañeda, Guarro \& Cano, Mycotaxon 58: 254. 1996. Figs. 6a y $6 \mathrm{~b}$.

Colonias efusas, pilosas, pardas oscuras. Conidióforos macronematosos, mononematosos, rectos o flexuosos, pardos en la base, más claros hacia el ápice, 10-25 $\times$ 3-3.5 $\mu \mathrm{m}$. Células conidiógenas poliblásticas, terminales, simpodiales, subhialinas, denticuladas. Conidios filiformes, sin septos, hialinos, 15-20 $\times 1 \mu \mathrm{m}$. 
Material estudiado. Hojas en descomposición. CHM-UJAT-07. Col. K. Martínez-Rivera, 11/02/2011.

Observaciones. Dactylaria filiformis es muy parecida a D. scolecospora (Kirk, 1983); se diferencian en que los conidios de la última son más largos y delga$\operatorname{dos}(30-45 \times 0.5-1)$.

Distribución. No se encontró algún registro posterior a la descripción de la especie, la cual fue detectada en material recolectado en Cuba (Castañeda-Ruiz et al., 1996).

Dactylaria hemibeltranioidea R.F. Castañeda \& W.B. Kendr., Univ. Waterloo Biol. Ser. 35: 27. 1991. Fig. 7.

Colonias efusas, pilosas, pardas oscuras. Conidióforos macronematosos, mononematosos, solitarios, erectos, rectos o flexuosos, simples, pardos en la base, más claros hacia el ápice, 40-75 × 3-3.5 $\mu \mathrm{m}$. Células conidiógenas poliblásticas, terminales, integradas, denticuladas. Conidios cilíndricos, hialinos, 7-9 $\times 2-2.3 \mu \mathrm{m}$.

Material estudiado. Ramas en descomposición. CHM-UJAT-08. Col. K. Martínez-Rivera, 11/11/2010.

Observaciones. La longitud de los conidios del material examinado es menor a la del ejemplar tipo (12-20.5 $\mu \mathrm{m})$, reportada por Castañeda-Ruiz y Kendrick (1991). Sin embargo, debido a que el resto de las características concuerdan con la especie, se decidió ubicar el material como $D$. hemibeltranioidea y ampliar el intervalo de las dimensiones de la longitud del conidio.

Distribución. Desde la descripción original de la especie en Cuba (Castañeda-Ruiz y Kendrick, 1991), solo ha sido reportada para Tailandia (Pinruan et al., 2007).

Dactylaria uniseptata Matsush., Microfungi of the Solomon Islands and Papua-New Guinea: 19. 1971. Fig. 8.

Colonias pilosas, pardas oscuras. Conidióforos macronematosos, mononematosos, simples, rectos o flexuosos, pardos oscuros en la base, más claros hacia el ápice, 50-98 × 3-3.5 $\mu \mathrm{m}$. Células conidiógenas poliblásticas, terminales, integradas, simpodiales, denticuladas, subhialinas. Conidios elipsoidales, truncados en la base, con un septo en la parte media, gutulados, subhialinos, 14-16 $\times$ 4-5 $\mu \mathrm{m}$.

Material estudiado. Ramas en descomposición. CHM-UJAT-09. Col. K. Martínez-Rivera, 11/02/2011. 
Distribución. Esta especie ha sido recolectada en el continente asiático en localidades de China (Cai et al., 2002 y Zhuang, 2001) así como en Nueva Guinea (Matsushima, 1971).

Melanocephala cupulifera S. Hughes, New Zealand J. Bot. 17: 168. 1979. Fig. 9.

Colonias dispersas, de pardas oscuras a negras. Conidióforos macronematosos, mononematosos, simples, erectos, rectos, pardos en la base, más claros hacia el ápice, lisos, $60-83 \times 6-7.5 \mu \mathrm{m}$. Células conidiógenas monoblásticas, integradas, con 3-6 proliferaciones percurrentes, caliciformes. Conidios solitarios, simples, obovoides, 2-3 septos transversales gruesos, pardo negruzcos, ligeramente constreñidos, base truncada y ápice redondeado, pardos oscuros, 33-39 × 19-23 $\mu \mathrm{m}$.

Material estudiado. Ramas en descomposición. CHM-UJAT-17. Col. K. Martínez-Rivera, 11/02/2011.

Observaciones. Los conidióforos del material estudiado son más pequeños que los reportados por Hughes (1979; 80-110 $\mu \mathrm{m})$. Sin embargo, el tamaño y forma de los conidios coinciden con los de la especie tipo (30-43 x 18-23.5 $\mu \mathrm{m})$ (Hughes, 1979).

Distribución. Esta especie ha sido reportada de Nueva Zelanda (Hughes, 1979 y McKenzie et al., 1992), Hong Kong (Zhuang, 2001) y la India (Rao y Hoog, 1986).

Monilochaetes guadalcanalensis (Matsush.) I.H. Rong \& W. Gams, Mycotaxon 76: 455. 2000. Fig. 10.

Colonias dispersas, pardas oscuras, pilosas. Conidióforos erectos, rectos o ligeramente flexuosos, septados, lisos, pardos oscuros en la parte basal, más claros hacia el ápice, 257-300 × 5-6 $\mu \mathrm{m}$, en la parte apical de 2-3 $\mu \mathrm{m}$. Células conidiógenas monofialídicas, con collarín terminal, percurrentes. Conidios catenulados, elipsoidales, lisos, parte basal truncada y parte apical redondeada, hialinos, 17-21 $\times 5-8$ $\mu \mathrm{m}$.

Material estudiado. En hojas en descomposición. CHM-UJAT-19. Col. K. Martínez-Rivera, 11/11/2010.

Observaciones. El ejemplar estudiado difiere ligeramente del material tipo en el ancho de los conidios $(16-30 \times 8-10 \mu \mathrm{m})$ (Matsushima, 1971). Sin embargo, el resto de la morfología coincide con la de la especie. La ubicación taxonómica de este hongo ha tenido varios cambios: Gams y Holubová-Jechová (1976) la transfirieron del género Catenularia a Exochalara, más tarde Rong y Gams (2000) la ubicaron nuevamente en el género Monilochaetes. 

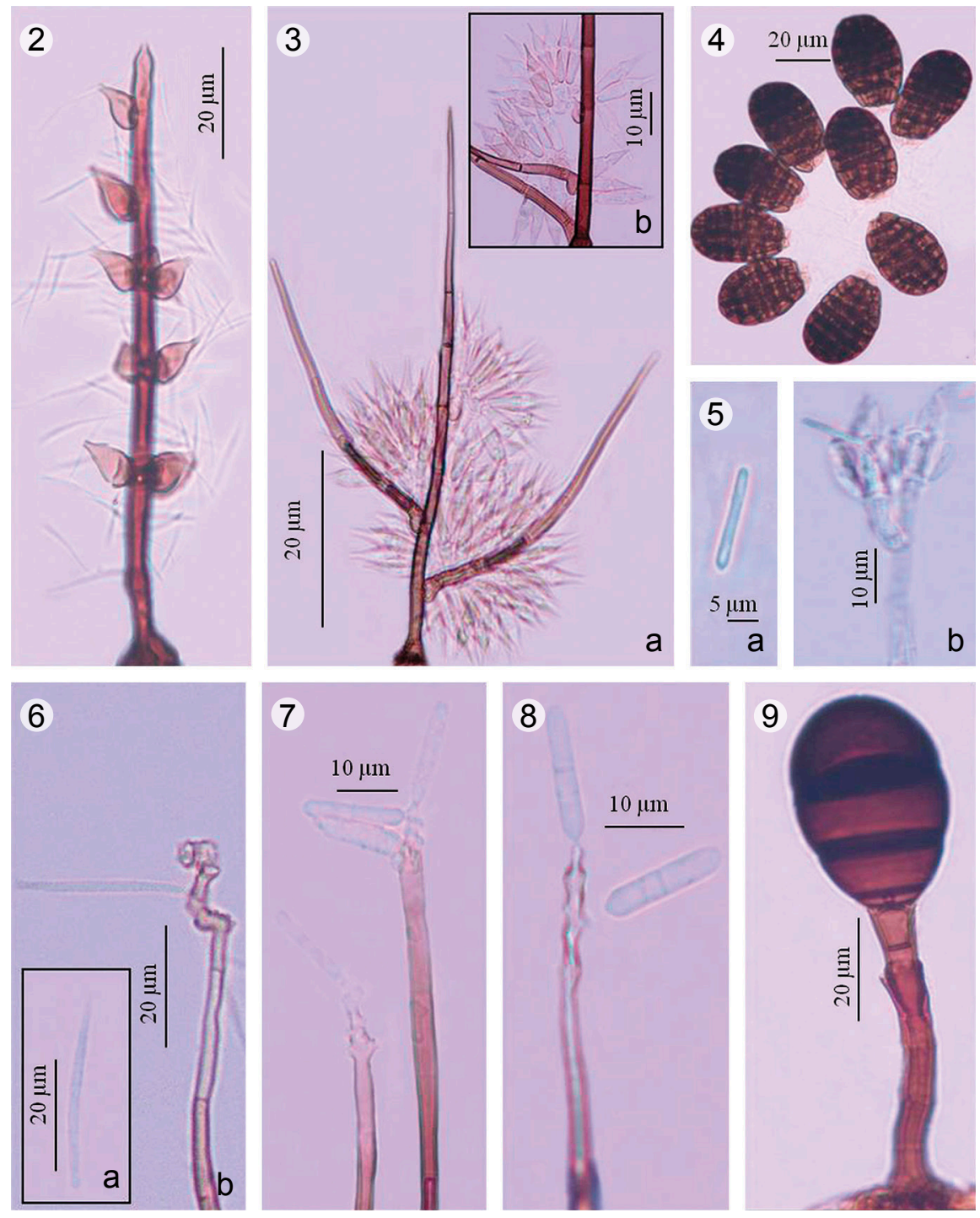

Figs. 2-9.2. Atrosetaphiale flagelliformis: conidióforo con células conidiógenas ampuliformes y conidios filiformes. 3. Beltraniopsis ramosa: a. conidióforo setiforme ramificado, b. células separadoras y conidios bicónicos. 4. Berkleasmium sinense: esporodoquio con conidios muriformes. 5. Clonostachys compactiuscula: a. conidio cilíndrico, b. parte apical del conidióforo peniciliforme. 6. D. filiformis: a. conidios filiformes, b. conidióforo con células conidiógenas denticuladas. 7. D. hemibeltranioidea: conidióforo con células conidiógenas simpodiales y conidios cilíndricos. 8. D. uniseptata: conidióforo con conidios elipsoidales con un septo transversal. 9. Melanocephala cupulifera: conidióforo con célula conidiógena caliciforme y conidio con dos septos. 
Distribución. Además del material tipo procedente del Japón (Matsushima, 1971), M. guadalcanalensis solo había sido recolectada en Cuba (Mercado-Sierra et al., 1997).

Mycoleptodiscus taiwanensis Matsush., Matsushima Mycol. Mem. 5: 21. 1987. Fig. 11.

Conidiomas de tipo esporodoquio, punctiformes, pardos oscuros, dispersos. Conidióforos ausentes. Células conidiógenas fialídicas, doliformes, 6-10.5 × 7-11 $\mu \mathrm{m}$. Conidios lunados, hialinos, $12-20 \times 6-6.5 \mu \mathrm{m}$, con una sétula en cada extremo de 1-3 $\mu \mathrm{m}$ de largo.

Material estudiado. Hojas en descomposición. CHM-UJAT-20. Col. K. Martínez-Rivera, 11/11/2010.

Observaciones. Mycoleptodiscus taiwanensis es muy similar a M. atromaculans y $M$. indicus, se diferencian en que los conidios de $M$. atromaculans son más largos $(16.5-25 \times 6.5-8.5 \mu \mathrm{m})$ y $M$. indicus presenta apéndices apicales de mayor tamaño $(1-10 \mu \mathrm{m})$.

Distribución. Solo se encontró el registro del material tipo, el cual fue detectado sobre raquis de hojas muertas de Areca catechu (Arecaceae) colectadas en Taiwán (Matsushima, 1987).

Sporidesmium dissolvens Hol.-Jech., Mercado \& J. Mena, Česká Mykol. 40: 156. 1986. Fig. 12.

Colonias efusas, pilosas, pardas oscuras. Conidióforos macronematosos, mononematosos, erectos, rectos o ligeramente flexuosos, pardos oscuros aclarándose hacia el ápice, $32-115 \times 4.5 \mu \mathrm{m}$. Células conidiógenas monoblásticas, integradas, terminales, percurrentes, cilíndricas, pardas oscuras. Conidios obclavados, obpiriformes, con 3 septos, la célula basal más grande que las otras, pardos oscuros, la célula apical subhialina, con una túnica mucilaginosa en la punta, 30-40 $\times 10-12 \mu \mathrm{m}$, parte basal de 3-4 $\mu \mathrm{m}$.

Material estudiado. Ramas en descomposición. CHM-UJAT-29. Col. K. Martínez-Rivera, 11/11/2010.

Observaciones. El rasgo característico de $S$. dissolvens es la presencia de una túnica mucilaginosa, subhialina en la parte apical del conidio.

Distribución. Esta especie solo ha sido registrada para la zona del Caribe, Cuba (Holubová-Jechová et al., 1986) y Federación de Indias Occidentales (Minter et al., 2001). 
Stilbella clavispora Seifert, Stud. Mycol. 27: 80. 1985. Figs. 13a y 13b.

Conidiomas de tipo sinema, conspicuos, blanquecinos, cilíndricos, capitados, simples, claviformes, blancos, ligeramente pardos al madurar, 350-400 $\times 25-59 \mu \mathrm{m}$. Conidióforos macronematosos, ramificados, biverticilados, hialinos, 15-25 × 2-2.5 $\mu \mathrm{m}$. Células conidiógenas fialídicas, terminales, verticiladas, cilíndricas, hialinas, $30 \times 2 \mu \mathrm{m}$. Conidios elipsoidales, claviformes, hialinos, sin septos, aglutinados en una masa mucilaginosa, 8-12 × 3-4.5 $\mu \mathrm{m}$.

Material estudiado. Ramas en descomposición. CHM-UJAT-32. Col. K. Martínez-Rivera, 11/02/2011.

Observaciones. Las características morfológicas y las dimensiones del material estudiado coinciden con las de la descripción de la especie.

Distribución. Se encontraron registros de Suecia (Seifert, K.A. 1985) y de Cuba (CYBERNOME).

Thozetella havanensis R.F. Castañeda, Rev. Jardín Bot. Nac. 5: 69. 1984. Fig. 14.

Conidiomas de tipo sinema, dispersos, mucosos en el ápice, pardos oscuros en la base y blanquecinos en el ápice. Conidióforos macronematosos, cilíndricos, ramificados en el ápice. Células conidiógenas fialídicas, cilíndricas, ampuliformes o lageniformes. Microaristas sigmoides, alantoides, lisas de la parte basal al centro y rugosas en la parte apical, subhialinas, 17-21 $\times 2-3 \mu \mathrm{m}$. Conidios falciformes, hialinos, 11-13 $\times 1-2 \mu \mathrm{m}$, con una sétula filiforme de 4-6 $\mu \mathrm{m}$ de longitud en cada extremo.

Material estudiado. Hojas en descomposición. CHM-UJAT-34. Col. K. Martínez-Rivera, 11/11/2010.

Observaciones. El material estudiado concuerda con la morfología y las medidas proporcionadas por Castañeda-Ruiz (1984).

Distribución. Se encontraron registros para Brasil (Grandi et al., 1995), Cuba (Castañeda-Ruiz, 1984; Mercado-Sierra et al., 1997; Delgado-Rodríguez y MenaPortales, 2004b), España (CYBERNOME) y Nigeria (Calduch et al., 2002).

Vermiculariopsiella parvula Nawawi, Kuthub. \& B. Sutton, Mycotaxon 37:177. 1990. Figs. 15a y 15 b.

Conidiomas de tipo esporodoquio, solitarios, pardos oscuros, con una masa blanquecina en el ápice, rodeados de setas. Setas erectas, rectas o ligeramente flexuosas, lisas, septadas, pared gruesa con las puntas agudas, pardas oscuras, 110- 
$300 \times 3-4 \mu \mathrm{m}$. Conidióforos cilíndricos, cortos, densamente agrupados, hialinos. Células conidiógenas monofialídicas, lageniformes, hialinas. Conidios cilíndricos, lisos, unicelulares, gutulados, con el ápice ligeramente curvo o puntiagudo, hialinos, agrupados en masas mucilaginosas blanquecinas, 11-12 $\times 2-3 \mu \mathrm{m}$.

Material estudiado. Hojas en descomposición. CHM-UJAT-35. Col. K. Martínez-Rivera, 11/11/2010.

Observaciones. Vermiculariopsiella parvula se diferencia de las demás especies del género por presentar una pequeña curvatura en la parte apical del conidio.

Distribución. No se encontraron registros de la especie después de que fue descrita a partir de material colectado en Malasia (Nawawi et al., 1990).

Zygosporium geminatum S. Hughes, Mycol. Pap. 44: 5. 1951. Figs. 16a y 16b.

Colonias compactas, pardas oscuras. Conidióforos mononematosos, macronematosos, erectos, simples, subulados hacia el ápice, pardos, 90-110 $\times 3-5 \mu \mathrm{m}$. Vesículas curvas, truncadas, cónicas en el ápice, pardas oscuras, 12-15 × 6-7 $\mu \mathrm{m}$. Células conidiógenas monoblásticas, discretas, determinadas, ampuliformes, ovoides, hialinas. Conidios elipsoidales, verrugosos, pardos, 20-30 × 8-10 $\mu \mathrm{m}$.

Material estudiado. Hojas en descomposición de Aechmea bracteata (Bromeliaceae). CHM-UJAT-40. Col. K. Martínez-Rivera, 11/11/2010.

Observaciones. La morfología y las medidas del material estudiado concuerdan con las proporcionadas por Hughes (1951).

Distribución. Ha sido reportada del continente africano: Ghana (Hughes, 1951) y Uganda (Ellis, 1971), y del americano: Venezuela (Castañeda-Ruiz et al., 2003).

Especies registradas previamente para México

Beltrania rhombica Penz., Michelia 2: 474. 1882.

Material estudiado: Hojas en descomposición. CHM-UJAT-02. Col. K. Martínez-Rivera, 11/11/2010. Registros anteriores: Tabasco (Becerra et al., 2007), Tamaulipas y Veracruz (Heredia, 1994).

Dactylaria parvispora (Preuss) de Hoog \& Arx, Kavaka 1: 58. 1973.

Material estudiado: Hojas en descomposición. CHM-UJAT-24. Col. K. Martínez-Rivera, 11/11/2010. Registros anteriores: Veracruz (Heredia et al., 1997). 

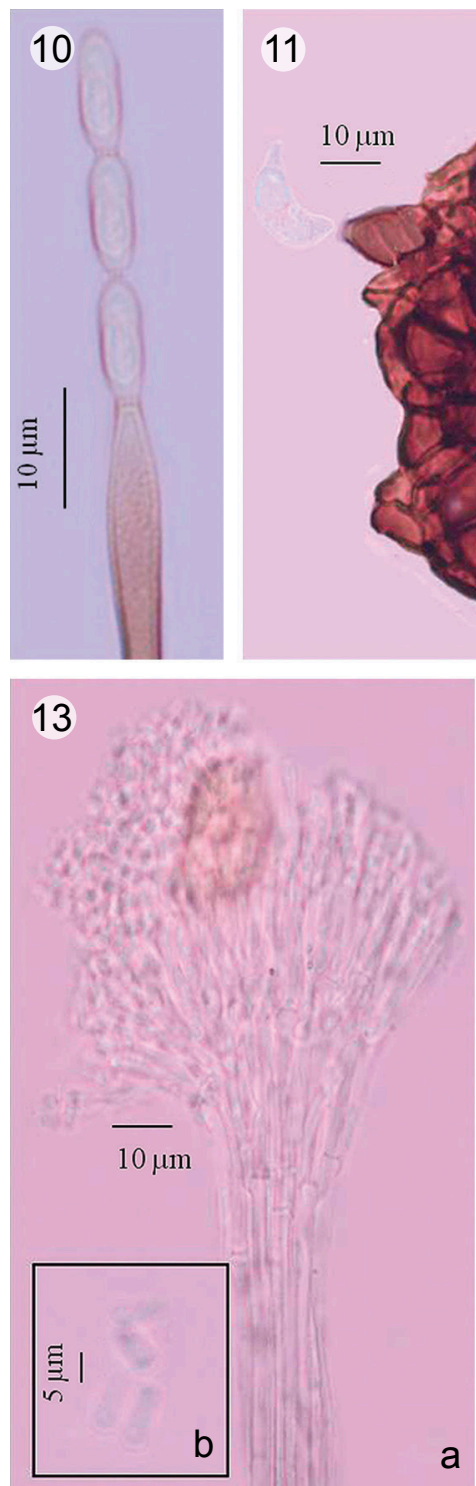
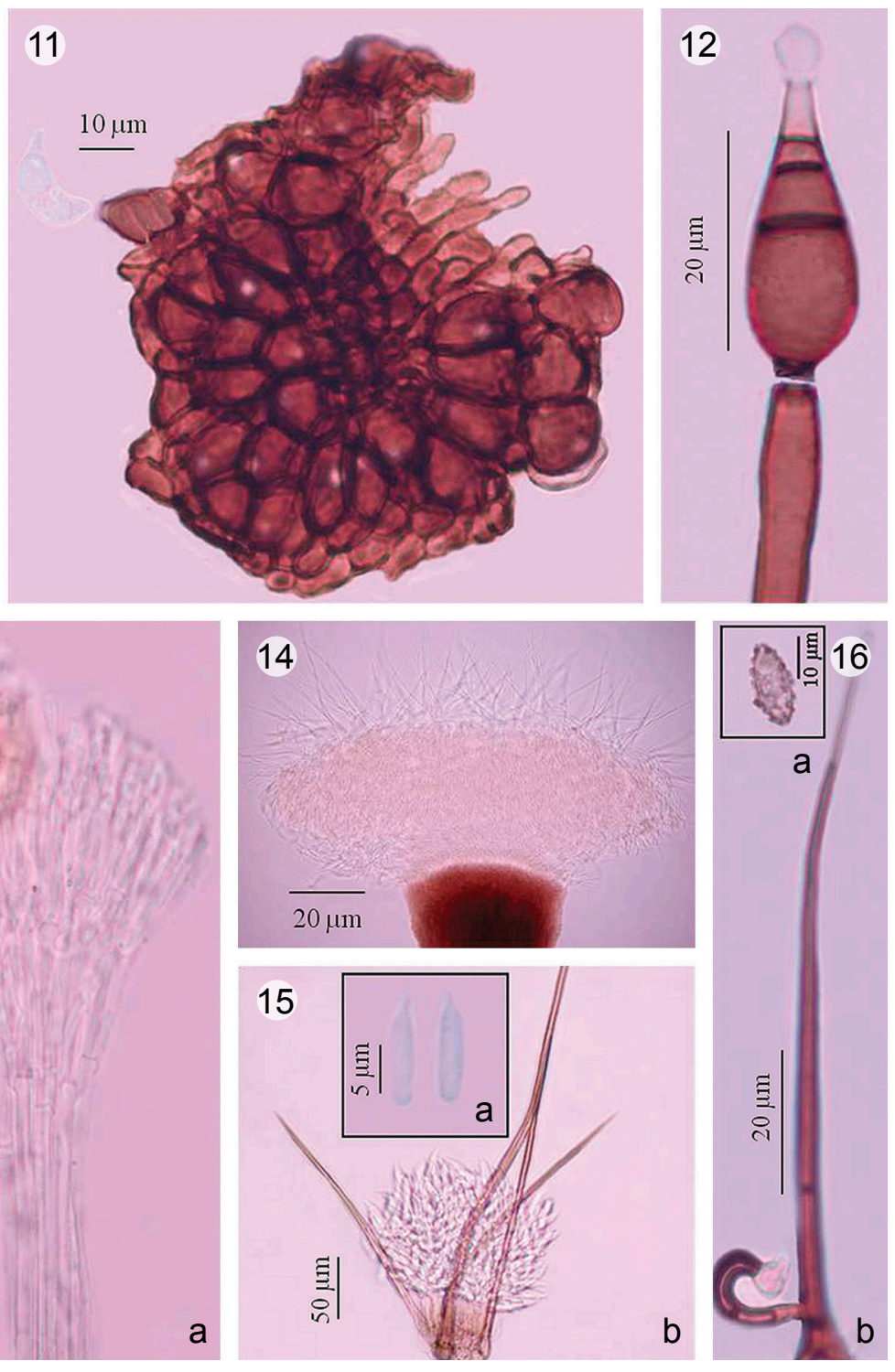

Figs. 10-16. 10. Monilochaetes guadalcanalensis: conidióforo con conidios catenulados. 11. Mycoleptodiscus taiwanensis: esporodoquio y conidio. 12. Sporidesmium dissolvens: conidióforo con conidio obclavado con gota mucilaginosa en el ápice. 13. Stilbella clavispora: a. parte apical del sinema con células conidiógenas y conidios, b. conidios claviformes. 14 . Thozetella havanensis: sinema con aristas y conidios. 15. Vermiculariopsiella parvula: a. conidios, b. esporodoquio con setas y conidios. 19. Zygosporium geminatum: a. conidio elipsoidal verrugoso, b. conidióforo con vesícula y célula conidiógena integrada. 
Dictyochaeta fertilis (S. Hughes \& W.B. Kendr.) Hol.-Jech., Folia Geobot. Phytotax. 19: 426. 1984.

Material estudiado: Ramas en descomposición. CHM-UJAT-10. Col. K. Martínez-Rivera, 11/11/2010. Registros anteriores: Veracruz (Heredia et al., 2000b).

Ellisembia adscendens (Berk.) Subram., Proc. Indian Natl. Sci. Acad. Part B. Biol. Sci. 58(4): 183. 1992.

Material estudiado: Ramas en descomposición. CHM-UJAT-11. Col. K. Martínez-Rivera, 11/11/2010. Registros anteriores: Tabasco (Becerra et al., 2007) y Veracruz (Heredia et al., 2004).

Exserticlava vasiformis (Matsush.) S. Hughes, New Zealand J. Bot. 16: 332. 1978.

Material estudiado: Hojas en descomposición de Aechmea bracteata (Bromeliaceae). CHM-UJAT-13. Col. K. Martínez-Rivera, 11/11/2010. Registros anteriores: Veracruz (Heredia et al., 2004).

Helminthosporium palmigenum Matsush., Microfungi of the Solomon Islands and Papua-New Guinea: p. 30. 1971.

Material estudiado: Ramas en descomposición. CHM-UJAT-14. Col. K. Martínez-Rivera, 11/11/2010. Registros anteriores: Tabasco (Becerra et al., 2007).

Hermatomyces amphisporus R.F. Castañeda \& Heredia, Cryptogamie Mycol. 21: 223. 2000.

Material estudiado: Ramas en descomposición. CHM-UJAT-15. Col. K. Martínez-Rivera, 11/02/2011. Registros anteriores: Veracruz (Castañeda-Ruiz y Heredia, 2000).

Junewangia globulosa (Tóth) W.A. Baker \& Morgan-Jones, Mycotaxon 81: 308. 2002.

Material estudiado: Ramas en descomposición. CHM-UJAT-16. Col. K. Martínez-Rivera, 11/11/2010. Registros anteriores: Tabasco (Becerra et al., 2007) y Veracruz (Heredia et al., 1997). 
Menisporopsis theobromae S. Hughes, Mycol. Pap. 48: 59. 1952.

Material estudiado: Hojas en descomposición. CHM-UJAT-18. Col. K. Martínez-Rivera, 11/11/2010. Registros anteriores: Tabasco (Heredia et al., 2006b), Tamaulipas (Heredia, 1994) y Veracruz (Heredia et al., 1997).

Periconia cookei E.W. Mason \& M.B. Ellis, Mycol. Pap. 56: 72. 1953.

Material estudiado: Hojas en descomposición. CHM-UJAT-21. Col. K. Martínez-Rivera, 11/11/2010. Registros anteriores: Veracruz (Arias et al., 2010).

Phaeoisaria clematidis (Fuckel) S. Hughes, Can. J. Bot. 36: 794. 1958.

Material estudiado: Ramas en descomposición. CHM-UJAT-22. Col. K. Martínez-Rivera, 11/02/2011. Registros anteriores: Tabasco (Heredia et al., 2006b) y Veracruz (Heredia et al., 1997).

Piricaudilium lobatum Hol.-Jech., Česká Mykol. 42: 200. 1988.

Material estudiado: Hojas en descomposición de Aechmea bracteata (Bromeliaceae). CHM-UJAT-23. Col. K. Martínez-Rivera, 11/02/2011. Registros anteriores: Veracruz (Heredia et al., 1997).

Septomyrothecium uniseptatum Matsush., Bull. Natn. Sci. Mus., Tokyo 14(3): 470. 1971.

Material estudiado: Hojas en descomposición. CHM-UJAT-25. Col. K. Martínez-Rivera, 11/11/2010. Registros anteriores: Tabasco (Heredia et al., 2006b).

Speiropsis hyalospora Subram. \& Lodha, Can. J. Bot. 42: 1062. 1964.

Material estudiado: Hojas en descomposición. CHM-UJAT-26. Col. K. Martínez-Rivera, 11/11/2010. Registros anteriores: Veracruz (Heredia et al., 2000a).

Sporidesmium amglicum (Grove) M.B. Ellis, Mycol. Pap. 70: 60. 1958.

Material estudiado: Ramas en descomposición. CHM-UJAT-28. Col. K. Martínez-Rivera, 11/11/2010. Registros anteriores: Veracruz (Heredia et al., 2006a). 
Sporidesmium tropicale M.B. Ellis, Mycol. Pap. 70: 58. 1958.

Material estudiado: Ramas en descomposición. CHM-UJAT-30. Col. K. Martínez-Rivera, 11/11/2010. Registros anteriores: Tabasco (Heredia et al., 2006b).

Subulispora longirostrata Nawawi \& Kuthub., Mycotaxon 30: 459. 1987.

Material estudiado: Hojas en descomposición. CHM-UJAT-33. Col. K. Martínez-Rivera, 11/11/2010. Registros anteriores: Veracruz (Heredia et al., 2004).

Veronaea coprophila (Subram. \& Lodha) M.B. Ellis, More Dematiaceous Hyphomycetes: p. 210. 1976.

Material estudiado: Hojas y ramas en descomposición. CHM-UJAT-36. Col. K. Martínez-Rivera, 11/11/2010. Registros anteriores: Tabasco (Becerra et al., 2008) y Veracruz (Heredia et al., 1995).

Virgatospora echinofibrosa Finley, Mycologia 59: 538. 1967.

Material estudiado: Hojas en descomposición. CHM-UJAT-37. Col. K. Martínez-Rivera, 11/11/2010. Registros anteriores: Veracruz (Heredia et al., 1997).

Wiesneriomyces laurinus (Tassi) P.M. Kirk, Trans. Br. Mycol. Soc. 82: 748. 1984.

Material estudiado: Hojas en descomposición. CHM-UJAT-38. Col. K. Martínez-Rivera, 11/11/2010. Registros anteriores: Tabasco (Becerra et al., 2007) y Veracruz (Heredia et al., 2000b).

Zygosporium echinosporum Bunting \& E.W. Mason, Mycol. Pap. 5: 135. 1941.

Material estudiado: Hojas en descomposición. CHM-UJAT-39. Col. K. Martínez-Rivera, 11/11/2010. Registros anteriores: Tabasco (Becerra et al., 2007) y Veracruz (Heredia et al., 1997).

Zygosporium gibbum (Sacc., M. Rousseau \& E. Bommer) S. Hughes, Can. J. Bot. 36: 825. 1958. 
Material estudiado: Hojas en descomposición de Aechmea bracteata (Bromeliaceae). CHM-UJAT-41. Col. K. Martínez-Rivera, 11/11/2010. Registros anteriores: Veracruz (Heredia y Reyes, 1999).

Zygosporium masonii S. Hughes, Mycol. Pap. 44: 15. 1951.

Material estudiado: Ramas en descomposición. CHM-UJAT-42. Col. K. Martínez-Rivera, 11/11/2010. Registros anteriores: Tabasco (Becerrra et al., 2007) y Veracruz (Heredia y Mercado-Sierra, 1998).

Zygosporium minus S. Hughes, Mycol. Pap. 44: 6. 1951.

Material estudiado: Hojas en descomposición de Aechmea bracteata (Bromeliaceae). CHM-UJAT-43. Col. K. Martínez-Rivera, 11/11/2010. Registros anteriores: Tabasco (Becerrra et al., 2007) y Veracruz (Heredia et al., 1997).

Zygosporium oscheoides Mont., Annls. Sci. Nat. Bot. ser. 2, 17: 121. 1842.

Material estudiado: Hojas en descomposición. CHM-UJAT-44. Col. K. Martínez-Rivera, 11/11/2010. Registros anteriores: Tabasco (Becerrra et al., 2007) y Veracruz (Mercado-Sierra y Heredia, 1994).

\section{AGRADECIMIENTOS}

Este trabajo se realizó mediante el apoyo de los proyectos FOMIX-CONACYT (TAB-2009-C18-122083) "Diversidad y conservación de los hongos macro y microscópicos saprobios de algunos ambientes del Parque Estatal Agua Blanca, Macuspana, Tabasco" y PROMEP (UJAT-PTC-094) "Evaluación de las comunidades de hongos microscópicos saprobios del suelo y de la hojarasca del Parque Estatal Agua Blanca, Macuspana, Tabasco". La primera autora agradece la beca que le fue otorgada a través del primer proyecto y al Consejo Nacional de Ciencia y Tecnología (CONACYT-320647) por el apoyo obtenido para los estudios de Maestría en Ciencias Ambientales de la Universidad Juárez Autónoma de Tabasco. Los autores reconocen las facilidades otorgadas por la UJAT y por el Instituto de Ecología, A.C. 


\section{LITERATURA CITADA}

Anónimo. 2009. Anuario estadístico del estado de Tabasco. Instituto Nacional de Estadística, Geografía e Informática. Gobierno del estado de Tabasco. Villahermosa, México. $707 \mathrm{pp}$.

Arambarri, A., M. N. Cabello y A. S. Mengascini. 1987. Estudio sistemático de los Hyphomycetes del Río Santiago. II. Bol. Soc. Argent. Bot. 25: 213-222.

Arias, R. M., G. Heredia y J. Mena-Portales. 2010. Adiciones al conocimiento de la diversidad de los hongos anamorfos del bosque mesófilo de montaña del estado de Veracruz. III. Acta Bot. Mex. 90: 19-42.

Barbosa, R. F., L. M. Costa y L. F. P. Gusmão. 2009. Novos registros de Hyphomycetes decompositores para o estado da Bahia, Brasil. Acta Bot. Bras. 23: 323-329.

Barnett, H. L. y B. B. Hunter. 1987. Illustrated genera of imperfect fungi. 4a ed. MacMillan Pub. Co. Nueva York, USA. 118 pp.

Becerra, C., G. Heredia y R. M. Arias. 2007. Contribución al conocimiento de los hongos anamorfos saprobios del estado de Tabasco. II. Rev. Mex. Micol. 24: 39-53.

Becerra, C., G. Heredia, R. M. Arias, J. Mena-Portales y R. F. Castañeda-Ruiz. 2008. Contribución al conocimiento de los hongos anamorfos saprobios del estado de Tabasco. III. Rev. Mex. Micol. 28: 25-39.

Becerra, C., G. Heredia, R. M. Arias, R. F. Castañeda-Ruiz y J. Mena-Portales. 2011. Especies raras de hongos anamorfos saprobios en el estado de Tabasco. Acta Bot. Mex. 96: 15-31.

Cai, L., C. K. M. Tsui, K. Zhang y K. D. Hyde. 2002. Aquatic fungi from Lake Fuxian, Yunnan, China. Fungal Divers. 9: 57-70.

Calduch, M., J. Gené, J. Guarro, A. Mercado-Sierra y R. F. Castañeda-Ruiz. 2002. Hyphomycetes from Nigerian rain forests. Mycologia 94: 127-135.

Capdet, M. y A. Romero. 2010. Fungi from palms in Argentina. Mycotaxon 112: 339-355.

Carmichael, J. W., W. B. Kendrick, I. L. Conners y L. Sigler. 1980. Genera of Hyphomycetes. The University of Alberta Press. Edmonton, Canadá. 388 pp.

Castañeda-Ruiz, R. F. 1984. Nuevos taxones de Deuteromycotina: Arnoldiella robusta gen. et sp. nov.: Roigiella lignicola gen. et sp. nov. Sporidesmium pseudolmediae sp. nov. y Thozetella havanensis sp. nov. Rev. Jard. Bot. Nac. 5(1): 57-87.

Castañeda-Ruiz, R. F. y G. Heredia. 2000. Two new dematiaceous Hyphomycetes on Cyathea from Mexico. Cryptogamie Mycol. 21: 221-228.

Castañeda-Ruiz, R. F. y W. B. Kendrick. 1991. Ninety-nine conidial fungi from Cuba and three from Canada. Univ. Waterloo Biol. Ser. 35: 1-132.

Castañeda-Ruiz, R. F., J. Guarro y J. Cano. 1996. Notes of conidial fungi. VIII. Two new species of Dactylaria from Cuba. Mycotaxon 58: 253-258.

Castañeda-Ruiz, R. F. y G. R. W. Arnold. 1985. Deuteromycotina de Cuba. I. Hyphomycetes. Rev. Jard. Bot. Nac. 6: 47-67.

Castañeda-Ruiz, R. F., J. Guarro, S. Velázquez-Noa y J. Géne. 2003. A new species of Minimelanolocus and some hyphomycete records from rain forests in Brazil. Mycotaxon 85: 231-239. 
Castañeda-Ruiz, R. F., T. Iturriaga, D. W. Minter, M. Saikawa, G. Vidal y S. VelazquezNoa. 2003. Microfungi from Venezuela, a new species of Brachydesmiella, a new combination, and new records. Mycotaxon 85: 211-229.

Castañeda-Ruiz, R. F., T. Iturriaga, D. W. Minter, G. Heredia-Abarca., M. Stadler, M. Saikawa y R. Fernández. 2009. Two new anamorphic fungi and some microfungi recorded from El Avila, Venezuela. Mycotaxon 107: 225-237.

Castillo, A. O. 1995. Los recursos maderables del estado de Tabasco. Informe técnico, Consejo Nacional de Ciencia y Tecnología. Convenio PC22089. Villahermosa, México. 220 pp.

Castillo, A. O. y C. J. Zavala. 1996. Fisiología, recursos vegetales y alternativas del manejo en el Parque Estatal Agua Blanca, Tabasco. Universidad y ciencia 12: 63-70.

Delgado-Rodríguez, G. y J. Mena-Portales. 2004a. Hifomicetos (hongos anamórficos) de la Reserva Ecológica "Alturas de Banao" (Cuba). Bol. Soc. Micol. Madrid 28: 115-124.

Delgado-Rodríguez, G. y J. Mena-Portales. 2004b. Hifomicetos aero-acuáticos e ingoldianos de la reserva de la biosfera Sierra del Rosario (Cuba). Bol. Soc. Micol. Madrid 28: $105-113$

Delgado-Rodríguez, G., J. Mena-Portales, M. Calduch y C. Decock. 2002. Hyphomycetes (hongos mitospóricos) del área protegida Mil Cumbres, Cuba Occidental. Cryptogamie Mycol. 23: 277-293.

Ellis, M. B. 1971. Dematiaceous Hyphomycetes. Commonwealth Mycological Institute. Kew, UK. 608 pp.

Ellis, M. B. 1976. More dematiaceous Hyphomycetes. Commonwealth Mycological Institute. Kew, UK. 507 pp.

Farr, D. F., y A. Y. Rossman. Fungal databases. Systematic Mycology and Microbiology Laboratory, Agricultural Research Service, United States Department of Agriculture. Revisado el 31 de Mayo, 2013. Disponible en: http://nt.ars-grin.gov/fungaldatabases/.

Gams, W. y V. Holubová-Jechová. 1976. Chloridium and some other dematiaceous Hyphomycetes growing on decaying wood. Stud. Mycol. 13: 1-97.

Grandi, R. A. P., A. C. Grandi y W. B. C. Delitti. 1995. Hyphomycetes sobre folhas em decomposição de Cedrela fissilis Vell. Hoehnea 22: 27-37.

Gusmão, L. F. P. y R. A. P. Grandi. 1996. Espécies do grupo Beltrania (Hyphomycetes) associadas a folhas de Cedrella fissilis Vell. (Meliaceae), em Maringá, PR, Brasil. Hoehnea 23: 91-102.

Gusmão, L. F. P., R. A. P. Grandi y A. I. Milanez. 2001. Hyphomycetes from leaf litter of Miconia cabussu in the Brazilian Atlantic rain forest. Mycotaxon 79: 201-213.

Hawksworth, D. L. y E. Punithalingam. 1975. New and interesting microfungi from Slapton, South Devonshire: Deuteromycotina II. Trans. Brit. Mycol. Soc. 64: 89-99.

Heredia, G. 1994. Hifomicetes dematiáceos en bosque mesófilo de montaña. Registros nuevos para México. Acta Bot. Mex. 27: 15-32.

Heredia, G. y A. Mercado-Sierra. 1998. Tropical Hyphomycetes of Mexico. III. Some species from the Calakmul Biosphere Reserve, Campeche. Mycotaxon 68: 137143.

Heredia, G. y M. Reyes. 1999. Hongos conidiales de bosque mesófilo: algunas especies folícolas y de la hojarasca desconocidas para México. Rev. Mex. Micol. 5: 79-88. 
Heredia, G., A. Mercado-Sierra y J. Mena-Portales. 1995. Conidial fungi from leaf litter in a mesophilic cloud forest of Veracruz, Mexico. Mycotaxon 55: 473-490.

Heredia, G., J. Mena-Portales, A. Mercado-Sierra y M. Reyes 1997. Tropical Hyphomycetes of Mexico II. Some species from the tropical biology station "Los Tuxtlas", Veracruz, Mexico. Mycotaxon 64: 203-223.

Heredia, G., R. M. Arias y M. Reyes. 2000a. Contribución al conocimiento de los hongos Hyphomycetes de México. Acta Bot. Mex. 51: 39-51.

Heredia, G., R. M. Arias y M. Reyes. 2000b. Leaf litter fungi. Eight setose conidial species unknown from Mexico. Rev. Mex. Micol. 16: 17-25.

Heredia, G., M. Reyes, R. M. Arias, J. Mena-Portales y A. Mercado-Sierra. 2004. Adiciones al conocimiento de la diversidad de los hongos conidiales del bosque mesófilo de montaña del estado de Veracruz. Acta Bot. Mex. 66: 1-22.

Heredia, G., R. M. Arias, J. Mena-Portales y A. Mercado-Sierra. 2006a. Adiciones al conocimiento de la diversidad de los hongos conidiales del bosque mesófilo de montaña del estado de Veracruz. II. Acta Bot. Mex. 77: 15-30.

Heredia, G., R. F. Castañeda-Ruiz, C. Becerra y R. M. Arias. 2006b. Contribución al conocimiento de los hongos anamorfos saprobios del estado de Tabasco. I. Rev. Mex. Micol. 23: 53-62.

Heredia, G., R. F. Castañeda-Ruiz y S. Cappello. 2008. Biología e importancia de los hongos microscópicos filamentosos. Heredia, G. (ed.). Tópicos sobre diversidad, ecología y uso de los hongos microscópicos. Programa Iberoamericano de Ciencia y Tecnología para el Desarrollo (CYTED) e Instituto de Ecología, A.C. Xalapa, Ver. México. pp. 7-26.

Holubová-Jechová, V., A. Mercado-Sierra y J. Mena-Portales. 1986. Studies on Hyphomycetes from Cuba IV. Dematiaceous Hyphomycetes from province Pinar del Rio. Ceská Mykol. 40(3): 142-164.

Hughes, S. J. 1951. Studies on micro-fungi X. Zygosporium. Mycol. Pap. 44: 1-18.

Hughes, S. J. 1979. Relocation of species of Endophragmia auct. with notes on relevant generic names. New Zealand J. Bot. 17: 139-188.

Kirk, P. M. 1983. New or interesting microfungi XI. Three new Hyphomycetes on Ulex europaeus. Trans. Br. Mycol. Soc. 81: 401-404.

McKenzie, E. H. C., P. J. O’Sullivan y J. P. Wilkie. 1992. A list of type specimens of New Zealand fungi held in DSIR Plant Protection Herbarium (PDD). Mycotaxon 43: 77156.

Matsushima, T. 1971. Microfungi of the Solomon Islands and Papua-New Guinea. Publicado por el autor. Kobe, Japón. 78 pp.

Matsushima, T. 1983. Matsushima Mycological Memories No. 3. Publicado por el autor. Kobe, Japón. 90 pp.

Matsushima, T. 1987. Matsushima Mycological Memories No. 5. Publicado por el autor. Kobe, Japón. 100 pp.

Matsushima, T. 1995. Matsushima Mycological Memories No. 8. Publicado por el autor. Kobe, Japón. 54 pp.

Matsushima, T. 1996. Matsushima Mycological Memories No. 9. Publicado por el autor. Kobe, Japón. 213 pp. 
Matsushima, T. 2001. Matsushima Mycological Memories No. 10. Publicado por el autor. Kobe, Japón. 214 pp.

Mercado-Sierra A. y G. Heredia. 1994. Hyphomycetes asociados a restos vegetales en el estado de Veracruz, México. Rev. Mex. Mic. 10: 33-48.

Mercado-Sierra, A. y J. Mena-Portales. 1995. Hifomicetes dematiáceos de tres provincias orientales de Cuba. Rev. Iberoam. Micol. 12: 101-107.

Mercado-Sierra, A., V. Holubová-Jechová y J. Mena-Portales. 1997. Monografie XXIII. Hifomicetes demaciáceos de Cuba. Enteroblásticos. Museo Regionale Di Scienze Naturali. Torino, Italia. $388 \mathrm{pp}$.

Minter, D., M. Rodríguez y J. Mena-Portales. 2001. Fungi of the Caribbean. An annotated checklist, PDMS Publishing. Londres, Inglaterra. 946 pp.

Nawawi, A., A. J. Kuthubutheen y B. C. Sutton. 1990. New species and combinations in Vermiculariopsiella (Hyphomycetes). Mycotaxon 37: 173-182.

Pinruan, U., K. D. Hyde, S. Lumyong, E. H. C. McKenzie y E. B. G. Jones. 2007. Occurrence of fungi on tissues of the peat swamp palm Licuala longicalycata. Fungal Divers. 25: 157-173.

Rao, V. y G. S. Hoog. 1986. New or critical Hyphomycetes from India. Stud. Mycol. 28: $1-84$.

Rong, I. H. y W. Gams. 2000. The Hyphomycete genera Exochalara and Monilochaetes. Mycotaxon 76: 451-462.

Rosique, G. J. E. y G. S. Cappello (en prensa). Diversidad de hongos. In: Mata, Z. E. y L. D. J. Palma (eds.). Biodiversidad en Tabasco: Estudio de estado. Comisión Nacional para el Conocimiento y Uso de la Biodiversidad. México, D.F., México.

Taylor, J. E. y K. D. Hyde. 2003. Microfungi of tropical and temperate palms. Fungal Divers. Res. Ser. 12: 302.

Zhuang, W. Y. (ed.). 2001. Higher fungi of tropical China. Mycotaxon, Ltd. Ithaca, USA. 485 pp. 
